\title{
Psychische Störungen und sozialwissenschaftliche Katastrophenforschung, 1949-1985
}

\author{
Cécile Stephanie Stehrenberger
}

\begin{abstract}
Mental IIIness and Social Science Disaster Research, 1949-1985
During the second half of the 20th century several American multidisciplinary social science „disaster research groups" conducted numerous field studies after earthquakes, factory explosions and "racial riots", both inside and outside of the United States. Their aim was to investigate the reactions and behavior of individuals, organizations and communities to disasters. All of these groups were either promoted or at least partly founded by different branches of the US military. This article will analyze the groups' studies and findings on the question of disasters' psychological effects. The main focus will be on the stance the scientists took on the diagnosis of psychological trauma - especially Post Traumatic Stress Disorder (PTSD) — and the post disaster therapeutic interventions that became widespread in the 1970s and early 1980s. The disaster researchers questioned the need for, as well as the effectiveness and efficiency of "disaster mental health". At the same time they advocated early ideas of resilience, which later became one of the most important concepts in disaster management and beyond.
\end{abstract}

Keywords: Disaster, disaster research, disaster mental health, trauma, resilience

Schlüsse/wörter: Katastrophen, Katastrophenforschung, Disaster Mental Health, Trauma, Resilienz

\section{Ein Wissensprodukionscluster, seine Ziele und Forschungsgegenstände}

„If I were to speculate about the future, I would forecast that the individual trauma approach will have relatively little standing within a decade. [...] In fact, there are signs that we are entering a transition stage from mythology to reality" (Quarantelli 1979: 22). Dies ist im Manuskript einer Rede zu lesen, die der Katastrophensoziologe und Mitbegründer des Forschungsfeldes disaster research Enrico Quarantelli im Jahr 1979 an der University of Cincinnati hielt. In einer überarbeiteten Version des Textes von 1984 gab er sich noch immer zuversichtlich, dass jener Übergang hin zu „Realität“ im Umgang mit 
psychischen Folgeschäden von Katastrophen bald stattfinden würde (Quarantelli 1984a, b: 2). Die Prognose, der individual trauma approach würde in sehr naher Zukunft nur noch auf geringe Zustimmung treffen, fehlt jedoch im neuen Manuskript. Dies überrascht wenig, da die Aufnahme der Post Traumatic Stress Disorder (PTSD)-Diagnose 1980 in das Diagnostic and Statistical Manual of Mental Disorders III (DSM-Klassifikationssystem) ein baldiges Ende des Traumabooms auch im Umgang mit Katastrophen in weite Ferne gerückt hatte.

Enrico Quarantelli war Mitglied der 1949 am National Opinion Research Center an der University of Chicago gegründeten Disaster Research Group. 1952 wurde ein Committee for Disaster Studies am National Research Council ins Leben gerufen und im Jahr 1963 eröffnete Quarantelli zusammen mit Kollegen das Disaster Research Center (DRC) an der Ohio State University. Diese zumindest teilweise armeefinanzierten Gruppen untersuchten von den 1950er bis in die 1980er Jahre in Feldstudien das menschliche Verhalten nach solchen Katastrophen wie Erdbeben, Fabrikbränden und racial riots. Aufgrund gemeinsamer Entstehungszusammenhänge und Forschungsansätze sowie personeller Kontinuitäten behandle ich die Gruppen im Folgenden als ein zusammenhängendes Cluster. Das Ziel dieses Clusters bestand, wie ich zu zeigen versuche, in der Etablierung eines praktischen Regulierungswissens, das Katastrophenschutzbeauftragte etwa in Fragen der Warnung und Evakuierung anleiten und so $\mathrm{zu}$ einer Optimierung des „Regierens von Bevölkerungen "1 in Ausnahmezuständen beitragen sollte. Die Leiter der verschiedenen Armeeabteilungen, die die Wissenschaftler/-innen finanzierten, hofften, dass sich aus den Studien über zivile Katastrophen Wissen ableiten lassen würde, das Verhaltensweisen in Kriegssituationen, insbesondere nuklearen Angriffen, prognostizierbar machen würde. Den Forscher/-innen selbst erschienen Katastrophen als ideale sozialwissenschaftliche Laboratorien, in denen die Essenzen menschlichen Verhaltens und die Grundzüge der Funktionsweise von Gesellschaft beobachtbar waren (vgl. ganzer Abschnitt: Stehrenberger 2014: 414).

Die psychischen Katastrophenreaktionen von Individuen und Gruppen stellten einen wichtigen Forschungsgegenstand des Clusters dar. Dabei standen die Probleme im Mittelpunkt, die „Schockzustände“ oder „Massenpanik“ für das Versorgen und Lenken der Bevölkerung mit sich bringen würden. In den 1970er Jahren - einem Zeitpunkt, zu dem sich die methodische Perspektive im Cluster von einer psychologischen hin $\mathrm{zu}$ einer soziologischen verschoben hatte - untersuchten die Forscher/-innen auch die ersten groß angelegten, staatlich finanzierten therapeutischen Katastropheninterventionen, die in den USA durchgeführt wurden. Ein besonderes Augenmerk lag dabei auf der organisationalen Funktionsweise von disaster mental health delivery systems. Die Forscher/-innen interessierten sich aber auch für die Frage, ob solche Interventionen überhaupt den eigentlichen Bedürfnissen der 
Katastrophenüberlebenden entsprachen. Diese Forschung und die Positionen zur Frage mentaler Folgeschäden von Katastrophen, die die Forscher/-innen über die Jahrzehnte hinweg einnahmen und vertraten, erörtere ich im vorliegenden Artikel.

Nach einem kurzen Überblick über die Historiographie der Traumadiagnose untersuche ich zuerst die Haltung, welche die sozialwissenschaftlichen Katastrophenforscher/-innen in den 1950er und 60er Jahren hinsichtlich der Frage mentaler Folgeschäden von Katastrophen einnahmen. Im Jahr 1974 verwüstete eine Wirbelsturmserie, der so genannte 1974 Super Outbreak, die Stadt Xenia im Bundesstaat Ohio. Mitglieder des DRC untersuchten die im Anschluss daran vorgenommene therapeutische Katastrophenintervention und verfassten dazu mehrere Studien. Diese Untersuchungen behandle ich im dritten Teil dieses Artikels. Die Katastrophenforscher/-innen stellten in ihrer Kritik am trauma approach die Notwendigkeit groß angelegter therapeutischer Katastropheninterventionen radikal in Frage, indem sie etwa anmerkten, dass diese auf Kosten der Deckung materieller Katastrophenbedürfnisse ging. Gleichzeitig ging es ihnen aber auch, wie ich ausführen werde, ganz wesentlich darum, in der Verhandlung der Trauma-Frage die Wissenschaftlichkeit ihres eigenen Feldes zur Geltung zu bringen. Schließlich stelle ich im letzten Teil dieses Textes die These auf, dass in den Positionen der Katastrophenwissenschaftler/-innen auch Grundannahmen über die Funktionsweise von Gesellschaften und Individuen zum Ausdruck kommen, die die Grundlage des Resilienzkonzepts darstellen, das später nicht nur im Feld des Katastrophenmanagements größte Bedeutung erlangte.

\section{Zur Historiographie der Traumadiagnose}

Heutzutage ist die Annahme, dass Opfer von Naturkatastrophen genauso wie die Opfer anderer Gewaltereignisse von diesen in jedem Fall „traumatisiert" werden ein „Gemeinplatz“ (Morris 2011: 264). In diesem Feld begann das „empire of trauma“ (Fassin and Rechtman 2009) seinen Siegeszug Anfang der 1970er Jahre. In verschiedenen Regionen der USA ereignete sich damals eine Reihe von Naturkatastrophen an Orten, an denen bereits community mental health Netzwerke existierten. Therapeut/-innen untersuchten und behandelten Katastrophenüberlebende in Kliniken und vor Ort in provisorischen Unterkünften. Ihre Einsätze wurden in den Massenkommunikationsmedien sehr positiv inszeniert, was zur parteienübergreifenden politischen Unterstützung für die Integration therapeutischer Maßnahmen in die staatlich organisierte Katastrophenhilfe beitrug (Morris 2011: 275-280). Den thera-

peutischen Interventionen folgten Nachuntersuchungen, in denen Psycholog/-innen, Soziolog/-innen und vor allem Psychiater/-innen sowohl 
die unmittelbaren als auch die längerfristigen psychischen Folgen der Ereignisse zu eruieren versuchten. Bei der Untersuchung der Folgen des Buffalo Creek-Dammbruchs im Jahr 1972 gehörte auch Robert Jay Lifton zum beteiligten Forscherteam, dessen Monographie zu den psychischen Kriegsschäden von Vietnamveteranen (Lifton 1973) einen Meilenstein für die Herausbildung des Konzepts der posttraumatischen Belastungsstörung (PTBS) darstellte. Diese Studien registrierten bei einer Vielzahl von Überlebenden Albträume, Depressionen, Psychosen sowie das sogenannte „survivor syndrom" (Schuldgefühle, überlebt zu haben) (vgl. Lifton \& Olson 1976). Im Disaster Relief Act von 1974 wurde in Sektion 143 schließlich erstmals eine Klausel integriert, die das National Institute of Mental Health (NIMH) autorisierte, nach Katastrophen psychologische Krisenhilfe anzubieten. Damit war der wohl bedeutendste Schritt hin zu einer Institutionalisierung von Disaster Mental Health gemacht. Im Verlauf der folgenden Dekaden kam es zu einem weiteren Ausbau von psychologischen Praxen in der amerikanischen Katastrophenhilfe (vgl. Neria et al. 2009), dem die sozialwissenschaftlichen Katastrophenforscher/-innen des hier untersuchten Clusters äußerst kritisch gegenüber standen.

Bereits für die 1970er Jahre sind erste wissenschaftsgeschichtliche Annäherungen an die Psychotraumatologie zu verzeichnen (Fischer-Homberger 1972). Seitdem ist ein beachtlicher Korpus an Forschungsliteratur entstanden, die sich damit auseinandersetzt, wie sich die Vorstellung herausbilden und durchsetzen konnte, dass Menschen von Gewaltereignissen zwingend schwerwiegende, längerfristige psychische Schäden davon tragen würden (vgl. zum Überblick u. a. Micale and Lerner 2001; Luckhurst 2013). Diese Literatur hat für verschiedene Länder die Herausbildung von Diagnosen wie railway spine, traumatische Neurose oder shell shock verfolgt, die in der zweiten Hälfte des 19. Jahrhunderts nach Eisenbahnunglücken und später nach den Weltkriegen auftauchten. ${ }^{2}$

In einer Gesamtschau haben Didier Fassin und Richard Rechtman in ihrer Genealogie des „empire of trauma“ gezeigt, dass dieses verschiedene Herkunftsstränge hat, die alles andere als linear verlaufen sind. Wissenschaftliche Errungenschaften wurden dabei ermöglicht oder zumindest begleitet von politischen und moralischen Verschiebungen, die in Bereichen wie der Wahrnehmung von Holocaustüberlebenden, Kriegsveteranen, Flüchtlingen und Opfern von Kindsmissbrauch zu einer zunehmenden Akzeptanz des Traumakonzepts führten (Fassin and Rechtman 2009).

Die wissenschaftsgeschichtliche Auseinandersetzung mit der Geschichte des Traumas hat deutlich gemacht, dass diese Geschichte nicht die der Entdeckung einer Krankheit, sondern die der Erfindung einer Diagnose ist und dass sie sich nicht als eine simple Fortschrittsgeschichte der Anerkennung menschlichen Leidens schreiben lässt. Dabei geht es, wie Allan Young und andere betonen, nicht darum, die Realität menschlicher Leidenserfahrungen 
zu negieren, sondern im Gegenteil darum, deren simplifizierende und nivellierende Kategorisierung in Diagnosen wie etwa der Posttraumatischen Belastungsstörung kritisch zu beleuchten (vgl. Young 1997; Kwon 2012). ${ }^{3}$

Andrew Morris hat in einem sehr instruktiven Überblick die Wissenschaftsgeschichte der Durchsetzung des Traumakonzepts im Umgang mit Natur- und Technikkatastrophen umrissen (Morris 2011). Viele Aspekte der von ihm präsentierten Erfolgsgeschichte des Traumas sollten weiter verfolgt werden, etwa die Verschränkung politischer Interessen und medialer Inszenierungsformen, die die Verbreitung des Traumadiskurses in diesem Feld vorangetrieben haben. Im vorliegenden Artikel gehe ich aber mit der Geschichte der sozialwissenschaftlichen Katastrophenforschung vielmehr Störelementen nach, die zeigen, dass das Traumakonzept nicht immer ohne Weiteres akzeptiert wurde, sondern auch Alternativen dazu formuliert wurden. Mein Text soll einen weiteren Beitrag zur kritischen Genealogie des Traumadiskurses im Sinne der vorgestellten Arbeiten von unter anderem Fassin, Rechtman und Morris liefern. Er soll diese um eine neue Perspektive erweitern, indem er die Geschichtlichkeit des Traumas mit Blick auf Forschungsfelder außerhalb von Medizin und Psychologie herausarbeitet.

\section{Die Katastrophenforschung und die Frage mentaler Folgeschäden nach Katastrophen in den 1950er und 1960er Jahren}

Die Frage, welche psychischen Reaktionen Katastrophen in den von ihnen betroffenen Menschen auslösen würden, war bereits zentral in den frühen Studien der Katastrophenforscher/-innen. Allerdings war dabei noch nicht die Rede von „Trauma“. Allgemein waren es meist keine klar umrissenen (diagnostischen) Konzepte, mit denen die Forscher/-innen operierten, wenn sie sich damit auseinandersetzten, ob Menschen nach Katastrophen „zusammenbrechen“, „hysterisch“ fliehen, vor „Schock“ „gelähmt“ oder für immer „seelisch krank“ würden. Das Fehlen einheitlicher, klar definierter Kategorien war auch der multidisziplinären Ausrichtung insbesondere der NRC disaster research group geschuldet. Diese dürfte in Teilen auch dafür verantwortlich gewesen sein, dass die Forschungsergebnisse, zu denen ihre Mitglieder bezüglich mentaler Katastrophenfolgen kamen, bisweilen in verschiedene Richtungen gingen.

Kurze Artikel in Zeitschriften wie dem Journal of Social Issues (Fritz \& Marks 1954) und The Annals of the American Academy of Political and Social Science (Fritz \& Williams 1957) fassten die Resultate verschiedener Studien zusammen. Diese Artikel negierten die Unterschiede in den Forschungsergebnissen zwar nicht gänzlich, vermittelten aber doch die Idee weitgehend 
einheitlicher Positionen der Gruppen. Der Einstieg in einen dieser Artikel liest sich folgendermaßen:

Sudden disaster strikes a community. How do human beings act in such a situation? According to a pervasive popular conception, they panic [...] losing all sense of concern for their fellow human beings. [...] Large numbers of people are left permanently deranged mentally. [...] Those experienced in actual disasters are able to reject this picture as a product of ignorance, inaccurate observation and fertile imagination. (Fritz and William 1957: 42)

Die Katastrophenforscher/-innen der 1950er Jahre brachten ihre Forschungsergebnisse als ein Gegenwissen $\mathrm{zu}$ "Katastrophenmythen“ vor, die insbesondere durch die Massenmedien verbreitet würden. Zu diesen Mythen, denen zufolge sich Menschen allgemein in und nach Katastrophen mehrheitlich ,irrational“",,anti-sozial“ und schlicht falsch verhalten würden, zählten sie auch die Vorstellung, Katastrophen würden bei den Überlebenden „permanente mentale Störungen“ auslösen. Der Umstand, dass so die Idee psychopathologischer Katastrophenreaktionen von den Forscher/-innen zum gleichen Mythenkomplex wie Vorstellungen anderer Formen devianten Benehmens gezählt wurde, verweist auf eine ganz bestimmte moralische Wertung Überlebender von Gewalterfahrungen. Wie Fassin und Rechtman (2009) nachgezeichnet haben, begann erst in den 1970er Jahren die seelische Versehrtheit dieser Überlebenden ihre Menschlichkeit zu bestätigen und ihren Opferstatus zu verifizieren. Noch bis in die 1950er und 60er Jahre hinein geriet wer von sich behauptete, von Gewalterfahrungen permanente und schwere mentale Schäden davongetragen zu haben, oft unter den latenten Verdacht sich asozial zu verhalten und die Krankheit nur zu simulieren beziehungsweise an einer Krankheit zu leiden, die allein das Resultat von Willensschwäche sei. Zu unterstellen, Überlebende von Katastrophen seien keineswegs zwangsläufig längerfristig psychisch versehrt, bedeutete vor diesem Hintergrund also, sie durch die Loslösung von jenem Verdacht als moralisch gute Subjekte zu verteidigen.

Indem sie die Frage mentaler Katastrophenfolgen an die devianten Verhaltens koppelt, macht die obige Quellenstelle auch deutlich, dass die Forscher/-innen nicht so sehr an der Frage mentaler Krankheiten per se interessiert waren, sondern vielmehr daran, welche Notwendigkeit sozialer Kontrolle sich aus den an sie gebundenen Verhaltensformen ergeben würde. So betonten sie in ihren Abgrenzungen zu „Katastrophenmythen“ auch, die „Aktivitäten“ von Menschen(gruppen) nach untersuchten Katastrophen seien „hoch organisiert" gewesen und „emotionale Reaktionen“ unter „rigider Kontrolle gehalten“ worden (Fritz and Marks 1954: 33).

Den guten Umgang, den Individuen mit Katastrophen an den Tag legten, brachten die Forscher/-innen auch damit in Zusammenhang, dass Kollektive, und allen voran „communities“ als Ganzes in und nach Katastrophensituationen ausgesprochen gut funktionieren, ja geradezu über sich hinauswachsen 
würden. Der Soziologe Charles Fritz sprach in einem 1961 erschienenen und seitdem vielzitierten Artikel von einem "amplified rebound effect" von Katastrophen:

The motivation of the actors within the system and the consequent total concentration of societal energy on the goals of survival and recovery usually result in the rapid reconstruction of the society and, beyond that, often produce a kind of "amplified rebound" effect, in which the society is carried beyond its pre-existing levels of integration, productivity, and capacity for growth. (Fritz 1961: 692)

Fritz und andere im Feld dominante Katastrophenforscher/-innen vertraten erstens die Ansicht, Affekte (z.B. Angst), die Menschen nach Katastrophen zwar kurzfristig befallen, aber keineswegs in schwere Krankheiten mündeten, seien durchweg nachvollziehbar. Sie würden sich nämlich in erster Linie aus einer berechtigten Sorge um die Angehörigen und die eigene materielle Zukunft speisen. Zweitens argumentierten Fritz und Kolleg/-innen, solchen Zuständen sei nicht mit Therapien, sondern nur durch Ursachenbekämpfung beizukommen. So schrieben sie: „Since so many symptoms arise out of such situational factors as fear for the safety of self and intimates, separation of family members [...] an effective ,treatment' lies in the alleviation of the situations which produced them" (Williams and Fritz 1957: 48).

Wie weiter unten noch deutlich werden wird, finden sich in den Forschungsmeinungen, die von Katastrophenforscher/-innen in den 1970er Jahren in der Traumadebatte vertreten wurden, augenfällige Kontinuitäten zu den hier beschriebenen Sichtweisen. Zunächst aber möchte ich auf einige Positionen in der frühen Katastrophenforschung zu sprechen kommen, die in den zuvor zitierten zusammenfassenden Artikeln nur am Rande auftauchen.

Es waren dies Perspektiven und Stimmen, denen zufolge mentale Folgeschäden nach Katastrophen durchaus verbreitet waren, dass jedoch vor allem bestimmte Menschen, die nach Katastrophen mit „emotionalen Problemen“ zu kämpfen hätten, dies nicht zugeben würden. So schrieben Moore und Friedsam in einer vom NRC finanzierten Studie: „It is also possible that there is a bias in Negro culture against admitting emotional difficulty; it may be that such a question aroused fear of being classed as ,crazy' with the possibility of institutionalization as a possible consequence" (Moore \& Friedsam, 1959: 136). Die ebenfalls vom NRC geförderte Martha Wolfenstein übte grundsätzliche Kritik daran, dass ihre Kolleg/-innen nicht hinter die kulturellen Normen entsprechende Fassade sehen würden, wenn Menschen von sich behaupteten, auf Katastrophen mit größter Selbstkontrolle reagiert zu haben. Sie schrieb: „Perhaps in America particularly, where emotional control is highly valued, and the possibility of experiencing depression or despair tends to be denied, the distress of the disaster victim, who behaves with such satisfactory outward restraint, is likely to be underestimated" (Wolfenstein 1957: 83 f.). Ihre Kolleg/-innen wiesen Wolfensteins Positionen zurück. Sie schlossen sie aus dem vom Cluster produzierten Wissenskomplex aus, indem sie in 
zusammenfassenden Artikeln nicht erwähnt, in Inventaren nicht aufgeführt oder später gar negativ auf sie Bezug genommen wurde (vgl. etwa Dynes and Quarantelli 1975: 124).

\section{Zur Entwicklung der Katastrophenforschung in den 1970er Jahren}

Vier Stunden nachdem am 4. April 1974 ein Tornado noch nie zuvor gemessenen Ausmaßes 33 Tote und 1.000 Verletzte in Xenia, Ohio hinterlassen hatte, waren die ersten Mitglieder des Disaster Research Centers vor Ort und verfolgten die Rettungsarbeiten. Zu diesen gehörte auch, dass etwa 80 Freiwillige - darunter Sozialarbeiter/-innen und Psycholog/-innen verschiedener mental health center aus der Region - Beratungstische installierten, an denen sie ihre Dienste zur Verfügung stellten. Da diese Tische von kaum jemandem aufgesucht wurden, gingen die Therapeut/-innen auch direkt auf die Überlebenden zu, um ihre Hilfe anzubieten.

Die Katastrophenforscher/-innen interviewten Therapeut/-innen und Überlebende, und zwar sowohl in den ersten Tagen nach dem Tornado als auch Monate später. Darüber hinaus führten sie eine schriftliche Umfrage in ausgewählten Haushalten durch. Die Wissenschaftler/-innen stellten in ihrer Studie nicht nur fest, dass es dem disaster mental health delivery system, das sie aus organisationssoziologischer und systemtheoretischer Perspektive analysierten, an Effektivität und Effizienz gemangelt habe. Vor allem konstatierten sie eine große Diskrepanz zwischen dem von den „Anbietern“ vermuteten und dem tatsächlich vorhandenen Bedarf an Krisenberatung. Ersterer basierte nämlich auf der Vorstellung, es müssten, wenn auch nicht kurzfristig, so doch zumindest langfristig, „irgendwelche“ mentalen Probleme auftauchen: „that there was ,something' to be concerned about was widely accepted", schrieb die Katastrophenforscherin Verta Taylor aus Quarantellis Team (Taylor 1976: 66). Diese Erwartungen sollten sich gemäß der $D R C$-Wissenschaftler/-innen keineswegs erfüllen. Sie hielten bezüglich des unmittelbaren Effekts des Tornados fest: „Overall, such evidence as does exist indicates that the immediate response of the emergency group and victim population in the impact area was, by most criteria, relatively reasonable, rational and responsible" (Taylor 1976: 52). Und weiter: „[...] there is practically no evidence of individual breakdown or personal hysteria the night of the tornado" (Taylor 1976: 51).

Was die psychische Langzeitwirkung der Katastrophe anbelangt, welcher die Forscher/-innen rund 18 Monate nach den Stürmen nachgingen, unterschieden sie zwischen mental illness, mental health problems und problems in living. Sie stellten bezüglich ersterer Kategorie fest, dass keine schweren, mit der Katastrophe in Verbindung stehenden Psychopathologien verzeichnet worden seien und Kliniken sogar einen Rückgang in der Nachfrage nach 
Therapien und Medikamenten vermerkt hätten. Hinsichtlich möglicher mental health problems registrierten die DRC-Mitarbeiter/-innen, dass ein relativ hoher Anteil der von ihnen befragten Personen angab, sich gelegentlich „depressed or low“ zu fühlen und an Schlafproblemen oder Appetitlosigkeit zu leiden. Diese Probleme wurden jedoch als geringfügig und kurzfristig klassifiziert. Nur wenige Menschen räumten angeblich ein, mehr Alkohol zu konsumieren. Scheidungs- und Suizidraten waren stabil geblieben, die Anzahl von Strafanzeigen hatte zu-, diejenige von tatsächlich registrierten Vergehen abgenommen. Vor allem aber bemerkten die Forscher/-innen, dass die Katastrophe auch geradezu „positive“ Effekte auf die emotionale Befindlichkeit vieler der von ihnen befragten Menschen gehabt habe:

A large percentage of the people reported extremely positive psychological reactions to the disaster event. For example, 84 percent of the population asserted that their tornado experience had shown them they could handle crises better than they once thought they could, while 69 percent responded that they felt they had met a great challenge and were better off for having met it. (Dynes and Quarantelli 1975: 228)

Was schließlich die problems in living, also die Schwierigkeiten in der Bewältigung des Alltags, der Nahrungsbeschaffung und der Unterbringung in Notunterkünften anging, so stellten die Forscher/-innen fest, dass diese Probleme die mit Abstand größte mentale Belastung für die Katastrophenüberlebenden darstellten.

In ihrer Monographie zur Wirbelsturmkatastrophe in Xenia betonten die Autor/-innen, ihre Forschungsergebnisse seien über die Untersuchung des konkreten Falles hinweg von Bedeutung. Entsprechend fühlten sich mehrere DRC-Mitglieder auch befugt, bei verschiedensten Gelegenheiten von ihrer Xenia-Studie ausgehend allgemeinere Kritik am trauma approach zu üben und Vorschläge über den richtigen Umgang mit katastrophenpsychologischen Bedürfnissen in Umlauf zu bringen (vgl. Tierney and Baisden 1977). Damit ist angesprochen, dass die Forscher/-innen insbesondere in ihren späteren Texten durchaus eingestanden, dass es nicht so sei ,that there always is no need for psychological or crisis counseling, or the delivery of mental health services at least in the broad sense of the term" (Quarantelli 1984a, b: 1). Sie hielten aber an der bereits in den 1950er Jahren vertretenen Ansicht fest, dass Katastrophen mehrheitlich keine schwerwiegenden psychopathologischen Störungen nach sich ziehen würden und dass disaster mental health nur einen Aspekt eines viele Bereiche umfassenden Hilfsprogramms nach Katastrophen darstellen sollte.

Die Katastrophenforscher/-innen betonten, dass insbesondere in den Augen der Katastrophenüberlebenden selbst therapeutische Katastrophenhilfe ein stark untergeordnetes Bedürfnis darstelle: „[P]eople themselves ranked their social service needs much higher than they did their mental health needs (Dynes and Quarantelli 1975: 237)." Weiter führten die DRC- 
Mitarbeiter/-innen an, eine prioritäre Ausrichtung der Katastrophenplanung auf mental health services sei deswegen problematisch, weil diese auf Kosten der Deckung von social services needs organisiert würde, worunter auch ganz grundlegende materielle Bedürfnisse wie warme Kleidung oder Verpflegung fielen. Dazu Quarantelli: „In an ideal situation with unlimited resources, I suppose all approaches could conceivably be used. But in a real world with finite resources, a more specific stance should be taken" (Quarantelli 1979: 23). Der Fokus der sozialwissenschaftlichen Katastrophenforschung auf die materiellen Bedürfnisse von Katastrophenüberlebenden kam nicht von ungefähr. In ihren Studien aus den 1960er und 1970er Jahren hatten sie festgestellt, dass diese Bedürfnisse beziehungsweise die Armut, in der die Katastrophenopfer lebten, nicht allein aus dem vorausgegangenen Desaster resultierte, sondern vielmehr eine ihrer Ursachen war. Die Katastrophenforscher/-innen stellten fest, dass die Armut, aber auch die Ethnizität oder das Geschlecht von Individuen oder Gemeinschaften einen wesentlichen Einfluss darauf hatte, ob Ereignisse für sie zu einer Katastrophe wurden oder nicht. Strukturelle Ungleichheiten hatten die Katastrophenforscher/-innen bereits als Krisenursache in ihren Untersuchungen der racial riots ermittelt, die in den 1960er Jahren in Watts, Detroit oder Newark ausgebrochen waren und die amerikanische Politik und Öffentlichkeit beunruhigten. Als dringend benötigte Maßnahmen, um einen drohenden Bürgerkrieg oder eine Revolution abzuwehren, schlugen sie die Inklusion von Afroamerikaner/-innen in Arbeits- und Wohnungsmärkte und einen "politischen Dialog“ (Dynes and Quarantelli 1968) vor, aber keine strukturellen Systemtransformationen.

\section{Die Wissenschaftlichkeit der Katastrophenforschung}

In diesen Positionen wird ersichtlich, dass die Forscher/-innen auch Naturkatastrophen als keine rein natürlichen, sondern vielmehr als soziale und politische Phänomene verstanden. Dass den Fürsprecher/-innen des trauma approaches dieses Verständnis abgehe, bemängelte Quarantelli in seiner bereits eingangs zitierten Rede: „The individual trauma approach still assumes that disaster victims respond primarily to the disaster agent or its immediate effect and have not yet fully seen that the surrounding social context is by far the more important factor" (Quarantelli 1979: 21). Und weiter:

There is what I would call a ,medical' view of the world which implies [...] that there is an objective reality out there responsible for pathologies of various kinds. On the other hand, there is what might be called the social problem' view of the world [...]. In the more extreme versions, adherents of this view argue that all disasters are politically defined events. (Quarantelli 1979: 20) 
Die Katastrophenforscher/-innen waren mit ihrer Sorge um das Politische beim Therapeutischen, das ihrer Ansicht nach in den Positionen und Praktiken der disaster mental health-Befürworter/-innen zu kurz kam, nicht allein: Bereits frühe Kritiker/-innen der amerikanischen Therapiekultur wie Christopher Lasch (1979) und Philipp Rieff (1966) beklagten, der „expressive Individualismus" und die extreme Selbstbezogenheit, auf die jene hinausliefe, habe eine Entpolitisierung der amerikanischen Gesellschaft zur Folge. Introspektion als oberstes Ziel resultiere demnach in einem Mangel an Aufmerksamkeit für andere und einer fehlenden Bereitschaft zu politischer Partizipation. ${ }^{4}$

Wie bereits ausgeführt, war für die Katastrophenforscher/-innen das Davontragen schwerer seelischer Verletzungen von einer Katastrophe weder Ausdruck von Normalität oder Menschlichkeit noch Beweis erlittener Leiden. Menschen wurde im Normalfall eine innere (moralische) Stärke zugeschrieben, die sie $\mathrm{zu}$ rational handelnden Subjekten machte, die weder Disziplinierung noch psychiatrischer Hilfe bedurften. Im Umkehrschluss führte das dazu, dass Personen, die von sich behaupteten, dieser Norm nicht zu entsprechen und infolgedessen Forderungen stellten/Hilfeleistungen beanspruchten, in den Augen der DRC-Mitglieder in Verdacht gerieten, dies aus unlauteren Motiven zu tun. So diskreditierte Quarantelli die im Anschluss an die Buffalo Creek-Flut erhobenen Daten, denen gemäß Betroffene schwerwiegende psychische Schäden davongetragen hatten, mit dem Verweis, die Erhebung sei im Zusammenhang mit einer Schadensersatzklage gemacht worden: „, [T] he reported observation from the Buffalo Creek disaster [...] were primarily obtained in connection with a law suit and thus are suspect in the eyes of many other researchers" (Quarantelli 1984a: 4). Das Misstrauen gegenüber Menschen, die wegen mentaler Folgeschäden von Gewaltereignissen finanzielle Anforderungen stellen/Wiedergutmachung einklagen, hat Tradition. Eine Tradition, die bis zum Konzept der Unfall- oder Begehrensneurose zurückreicht, das deutsche und französische Psychiater seit Ende des 19. Jahrhunderts entwickelt hatten, um die Krankheitssymptome zu erklären, welche Opfer von Unfällen - später auch Kriegen - angeblich entwickelten oder simulierten, weil sie die Aussicht auf Entschädigung vor Augen hatten (vgl. Fassin and Rechtman 2009: 37 f.).

Es war aber nicht nur ihre Verstrickung in Entschädigungsfragen, welche die Fürsprecher/-innen des trauma approach in den Augen der Katastrophenforscher/-innen suspekt machte, sondern auch ihre angeblich mangelnde Wissenschaftlichkeit. So kritisierte Quarantelli in seiner bereits mehrfach zitierten Rede 1979 in Cincinnati:

Let us now turn from beliefs to actualities. [....] How correct is the theoretical view in the mental health area that disasters are necessarily pervasive and deeply traumatic events? [....] What is the actual evidence? By that I mean what do we have that goes beyond isolated anecdotal examples or an occasional clinical 
impression? What findings and observations do we have which have been obtained according to standard scientific research procedures and analyses? (Quarantelli 1979: 12)

Damit verortete der Soziologe die Positionen des trauma approach im Bereich der Überzeugungen und Anekdoten statt in der Wirklichkeit. Schließlich fiel in seiner Rede auch der bereits in den 1950er Jahren von den Katastrophensoziolog/-innen verwendete Begriff des Mythologischen: „In my judgment, the individual trauma approach is still at the mythological stage that most of the social and behavioral disaster researchers were at about two decades ago" (Quarantelli 1979: 21). Quarantelli klassifizierte so den trauma approach gegenüber der sozialwissenschaftlichen Katastrophenforschung als rückständig. Deren Geschichte konstruierte er in einem Fortschrittsnarrativ: Nachdem sie anfangs irrtümlicherweise ihrerseits von den mythologischen Bildern panischer und halb verrückter Katastrophenopfer ausgegangen seien, hätten die Forscher/-innen schnell entdeckt, dass diese Bilder nicht den Tatsachen entsprächen. Die Vergangenheit des Clusters, die, wie oben beschrieben, keineswegs eine einheitliche Bewegung aller Beteiligten in eine bestimmte Richtung gewesen war, wurde dabei begradigt, um sie mobilisierbar $\mathrm{zu}$ machen. Das Sprechen über das Trauma stellte für die Katastrophenforscher/ -innen eine ideale Plattform dar, um ihre Wissenschaftlichkeit zu inszenieren und es scheint, dass ihnen diese Inszenierung annähernd so wichtig war wie die Traumafrage selbst.

\section{Die Katastrophenforschung und das Problem der Resilienz}

Wie bereits Quarantelli (1979) prognostizieren auch heute manche Autor/-innen ein baldiges Ende der Hochkonjunktur von Traumamodellen. Alison Howell etwa konstatiert für den militärischen und den Bereich der humanitären Hilfe eine Verschiebung der „Autorität“ des PTBS-Konzeptes hin zu demjenigen der Resilienz (2012: 214). Resilienz bezeichnet die psychische Fähigkeit eines Systems, Stresserfahrungen ohne bleibende Schäden zu bewältigen beziehungsweise sich schnell von ihnen zu erholen. Der inflationären Diagnose der PTBS, mit der in den vergangenen Jahrzehnten ganze Bevölkerungen nach Katastrophen fast schon pauschal belegt und entsprechend behandelt worden sind, setzen Katastrophenhelfer/-innen heute gemäß Howell vermehrt die Identifizierung und Förderung der Ressourcen der Überlebenden und die Hilfe zur psychologischen Selbsthilfe gegenüber (vgl. Howell 2012: 219 f.).

Der Begriff der Resilienz stammt aus der Werkstoffmechanik des 19. Jahrhunderts, wo er sich auf die Elastizität von Stoffen bezog (vgl. Höhler 2014: 431 f.). Herkunftsstränge der Idee lassen sich in unterschiedlichsten Feldern 
lokalisieren; so etwa in der Rede von „widerständigen Gehirnen“, welche die Strapazen des Ersten Weltkriegs unbeschadet überstanden (vgl. Kaufmann 1999) hätten. Die ersten systematischen, konzeptuellen Verwendungen von "Resilienz" in der psychologischen Forschung sind allerdings erst seit den 1950er Jahren zu verzeichnen (vgl. Höhler 2014: 434). Eine bedeutende Rolle spielt die Idee der Resilienz auch in der Ökologie, wo Crawford Stanly Holling und andere die Kapazität von Ökosystemen untersuchten, durch Anpassung und Stabilität auch größte Veränderungen aufzufangen und weiterzubestehen (vgl. Höhler 2014: 430). Nach der Jahrtausendwende wurde Resilienz schließlich auch im Umgang mit Naturkatastrophen und anderen gesellschaftlichen Krisen zum Referenzpunkt schlechthin (vgl. Brassett et al. 2013: 222). Dabei liegt der Fokus vor allem auf der Veränderungsfähigkeit von Systemen und ihrer Eigenschaft aus Katastrophen zu lernen und an ihnen zu wachsen (vgl. Höhler 2014: 439 f.; Promberger et al. 2015: 286 f.; Evans and Reid 2014: 30).

Wenn auch in der sozialwissenschaftlichen Katastrophenforschung die Verwendung des Resilienzbegriffs bis in die 1980er Jahre hinein eine absolute Ausnahme darstellte, ist es dennoch zutreffend, die Katastrophenforschung, wie Bonß dies in einem sporadischen Verweis tut, als „Vorläufer“ (Bonß 2015: 23) der Resilienzforschung zu bezeichnen. Tatsächlich steckte hinter der Katastrophenforschung bereits in den 1950er Jahren dasselbe grundsätzliche Erkenntnisinteresse, das auch die Beschäftigung mit Resilienz motiviert: Die Katastrophenforschung war wie andere - militärisch finanzierte - Forschungsfelder des Kalten Krieges, die sich mit der nuklearen Bedrohung auseinandersetzten, damit befasst, das letztendlich Unplanbare planbar zu machen (vgl. Eden 2003). Manche Generäle und Politiker hielten die Verhaltensweisen der Amerikaner/-innen bei einem nuklearen Angriff für so wenig kalkulierbar, dass es ihnen plausibel erschien, Erkenntnisse aus dem Studium von Naturkatastrophen als Annäherung zu verwenden. Bereits die frühen Katastrophenforscher/-innen des NRC glaubten dabei, die Kumulierung von möglichst viel Wissen über einzelne Katastrophenfälle würde es erlauben, ein Katastrophenmodell anzufertigen, das sich auf extreme Ereignisse aller Art (inklusive nuklearer Angriffe) übertragen ließe (vgl. Stehrenberger 2016). Diese Generalisierung sollte so die grundlegende Unsicherheit, von der im Zusammenhang mit Katastrophen immer auszugehen war, auffangen und es ermöglichen Pläne herzustellen, um Menschen darauf vorzubereiten, sich an jede nur denkbare und undenkbare Situation anzupassen. Genau darum geht es auch in der Resilienzforschung und -politik (vgl. Aradau 2014: 75, Höhler 2014: 442, Bonß 2015: 23 f.). Vor allem aber setzt das Resilienzkonzept auf die permanente Bereitschaft von Systemen, die auf jede Situation reagieren können (vgl. Höhler 2014: 442; Neocleous 2012). Preparedness als Leitmotiv ist indes kein neues Phänomen. Vor allem in der Zivilverteidigung (vgl. Davis 2007) und auch der militärischen Planung von 
vital security systems (vgl. Collier 2015) war sie während des Kalten Krieges oberstes Ziel. Angesichts der Tatsache, dass sie vom Office of Civil Defense mitfinanziert wurde, überrascht es wenig, dass sich auch die sozialwissenschaftliche Katastrophenforschung daran orientierte.

Was aber vor allem für einen Einbezug der Katastrophenforschung in die Genealogie des Resilienzkonzepts spricht, ist dass das Denken der Katastrophenforscher/-innen schon früh Ausdruck von und Plädoyer für genau jene Form des „Regierens von Bevölkerungen“ war, die später für die Entwicklung des Resilienzkonzepts maßgebend sein sollte. Ich lese die Befunde der Katastrophenforscher/-innen der 1950er Jahre, wonach sich Menschen von sich aus „sehr gut“ in Katastrophensituationen verhalten würden, als Appell an die planenden Katastrophenschutzbehörden, Bevölkerungen in Katastrophensituationen nicht in allererster Linie mit disziplinarischen Technologien zu regulieren. Sie stehen vielmehr für eine Gouvernementalität, die auf subtileren Regierungstechniken basiert und auf eine moralische Ökonomie der Eigenverantwortung setzt. Es ist kein Zufall, dass es bei der Regenerationsfähigkeit, welche die $D R C$-Mitglieder katastrophenerprobten Menschen zuschrieben, in erster Linie um die Erhaltung und Steigerung von Produktivität ging. Dies wird beispielsweise anhand der Verhaltensparameter deutlich, die die Forscher/ -innen zur Bewertung von Regenerationsfähigkeit aufführten. Dazu zählte, wie viele Tage die Überlebenden der Arbeit ferngeblieben waren, ob Alkoholismus und Kriminalität zugenommen hatten, aber auch wie gut sich Menschen in ihren Beziehungen und insbesondere in ihren Eheverhältnissen fühlten (vgl. Quarantelli 1984a: 6). Die letztgenannten Punkte signalisieren, dass die wieder oder erst neu erlangte Stärke von Individuen auch an ihrer Fähigkeit zu (heteronormativer) Konformität gemessen wurde. Diese Konformität wiederum war in jener Gouvernementalität Möglichkeitsbedingung der kapitalistischen Steigerungslogik (vgl. Dörre et al. 2009), für die Katastrophen letztendlich primär Katalysatoren von persönlichem und gesellschaftlichem/ökonomischem Wachstum waren. Da in der kritischen Auseinandersetzung mit dem Resilienzdiskurs dieser immer wieder als Ausdruck einer neoliberalen Ideologie bezeichnet wird (vgl. auch Evans and Reid 2014), sei an dieser Stelle betont, dass jene Steigerungslogik grundlegender als der Neoliberalismus ist.

Selbstverständlich sind die Kritik an der universellen Verbreitung von Traumadiagnosen - insbesondere der ausufernden Anwendung von PTBS -, die dagegen etwa die materiellen Bedürfnisse von Katastrophenüberlebenden betont, und das sich zunehmend verbreitende Resilienzkonzept nicht zwei Seiten derselben Medaille. Dass aber, wie das Beispiel der sozialwissenschaftlichen Katastrophenforschung zeigt, Herkunftsorte von beiden unter einem Dach existieren konnten, stimmt nachdenklich. Eine zentrale Voraussetzung für diese Koexistenz liegt meines Erachtens genau in der genannten Steigerungslogik und dem Fokus auf Eigenverantwortung, die im Falle der Katastrophenforschung beide genealogischen Linien begleitete, aber 
bekanntermaßen auch sonst nicht selten auszumachen ist. Genau dort, wo der trauma approach mit dem Verweis auf „wirkliche“, materielle Bedürfnisse zurückgewiesen wird, ohne dass gleichzeitig strukturelle soziale und ökonomische Transformationen eingefordert werden, kann gleichzeitig die tief angelegte Fähigkeit von Systemen betont werden, Krisen aus sich selbst heraus aufzufangen und an ihnen zu wachsen.

\section{Fazit Historisierung und Politisierung}

Als sich Anfang der 1970er Jahre der Traumadiskurs im Bereich der Katastrophenbewältigung $\mathrm{zu}$ verbreiten begann, beschlossen die Katastrophensoziolog/-innen des $D R C$, dieses Phänomen empirisch zu untersuchen. Der groß angelegten therapeutischen Katastrophenintervention, die 1974 auf den Tornado in der Stadt Xenia folgte, widmeten sie eine mehrmonatige Feldstudie. Die Forscher/-innen kamen dabei zu dem Schluss, dass Psychotherapien nicht den eigentlichen Bedürfnissen der notleidenden Bevölkerung entsprächen. Menschen hätten zum einen dringender einer Unterkunft, Nahrung und Kleider bedurft. Zum anderen seien sie auch keineswegs seelisch derart angeschlagen gewesen, wie dies die Befürworter/-innen des trauma approach behaupteten. Mentale Probleme seien zwar vorgekommen, jedoch nicht schwerwiegend und auch nicht langanhaltend gewesen. Vielmehr sei es vielen Individuen nach der Katastrophe im Wissen darum, diese gut gemeistert zu haben, sogar besser gegangen als zuvor. Seit den 1950er Jahren brachten die Forscher/-innen diese Erkenntnisse auch als Gegenwissen gegen die Katastrophenmythen der Massenpanik vor und inszenierten dabei ihre Wissenschaftlichkeit.

Die Haltung, die die Katastrophenforscher/-innen in der Traumafrage einnahmen, verweist auf Subjektkonzeptionen und Vorstellungen vom idealen Regieren von Bevölkerungen, denen zufolge Menschen als rationale und eigenverantwortliche Wesen gelten, die nicht in erster Linie über disziplinarische Praxen zu regulieren sind. Solche Grundannahmen stecken auch hinter dem Resilienzdenken, das heute den Umgang mit Katastrophen und Krisen prägt.

Die Vorstellung, dass gewaltsame Ereignisse ihre Opfer zwangsläufig traumatisieren würden, setzte sich nicht linear durch. Das hier untersuchte Beispiel der sozialwissenschaftlichen Katastrophenforschung verdeutlicht zum einen die Widerstände, die dem Traumakonzept entgegengebracht wurden. Darüber hinaus erweitert seine Untersuchung die Genealogie des Resilienzkonzepts um einen weiteren Herkunftsstrang. Die Historisierung von Trauma und Resilienz soll zur Entnaturalisierung von beiden beitragen. Wie 
der Blick auf die Katastrophenforschung gezeigt hat, sind dabei vor allem auch deren politische Möglichkeitsbedingungen zu betonen. Es sind gerade auch letztere, welche im Umgang mit Trauma und Resilienz - auch in deren Verwendung als analytische Werkzeuge in der historischen Untersuchung gewaltsamer Ereignisse - zu Vorsicht gemahnen.

\section{Anmerkungen}

1 Ich verwende den Begriff des „Regierens der Bevölkerung“ im Sinne Foucaults als ein Lenken von Individuen und Kollektiven durch verschiedene Institutionen (u. a. Staat, Kirche und Familie) und eine Kombination verschiedener Machttechnologien wie Disziplin, Biopolitik und Selbsttechnologien, vgl. Foucault 2004a, b; Foucault 1994.

2 Vgl. Harrington 2003; Eckhart 2005; Hofer 2012; Thomas 2009; Koch 2014; Lerner 1996, 2003; Goltermann 2009; Withuis 2010; Winter 2000; Shepard 2000; Leys 2000.

3 Darin besteht auch ein Anliegen der nicht-historischen Forschung, die sich seit den 1990er Jahren aus anthropologischer, politikwissenschaftlicher, aber auch psychologischer Perspektive kritisch mit der gegenwärtigen Anwendung der Traumadiagnose auseinandersetzt. Gerade die globale Anwendung der PTBS-Diagnose in der Beurteilung menschlicher Reaktionen auf Ereignisse nahezu sämtlicher Art in allen Teilen der Welt wird dabei als äußerst problematisch beurteilt. Dies geschieht unter anderem mit dem Verweis, sie führe zu einer Pathologisierung der Betroffenen und zu einer Medikalisierung menschlicher Erinnerung und komme einer neo-kolonialen Geste gleich (vgl. Summerfield 2001; Pupavac 2001; Bracken and Petty 1998). Neben der Zuschreibung individueller Traumata haben Autor/-innen auch diejenige kollektiver „historischer Traumata“ etwa in Folge von Genoziden beanstandet (vgl. Maxwell 2014). Schließlich sei am Rande erwähnt, dass mittlerweile auch zahlreiche Studien vorliegen, die die Rückprojektion der Traumadiagnose auf historische Gewalterfahrungen auch mit Bezug auf Europa und insbesondere den Zweiten Weltkrieg, kritisch beleuchten (vgl. Ackermann 2004; Schmitz and Seidel Arpaci 2011).

4 In den 1980er und 90er Jahren diagnostizierten auch Autor/-innen wie Bellah et al. (1985) und Rose (1989), der "Triumph der Therapie“ habe dazu geführt, dass das Soziale als Individualpsychologisches problematisiert, dem Verantwortungsbereich individueller Selbsttechnologien zugeschrieben und kollektiv-politischer Aspirationen entzogen würde. Seit einigen Jahren schreiben auch manche Kritiker/-innen des Trauma-Diskurses in verschiedenen Feldern diesem zu, Gewalt, Krieg und Armut zu pathologisieren und dadurch zu entpolitisieren. Dies geschehe etwa in der Teufelskreisargumentation, in der durch Gewalt und Armut ausgelöste Traumata als Ursache neuer Gewalt/Armut interpretiert werden (vgl. Summerfield 2002: 1105; vgl. zusammenfassend: Howell 2012: 217).

\section{Literatur}

Ackermann, Volker 2004. Das Schweigen der Flüchtlingskinder. Psychische Folgen von Krieg, Flucht und Vertreibung bei den Deutschen nach 1945. Geschichte und Gesellschaft (30): 434464.

Aradau, Claudia 2014. The Promise of Security. Resilience, Surprise and Epistemic Politics. Resilience (2.2): 73-87.

Bellah, Robert N. et al. 1985. Habits of the Heart. Individualism and Commitment in American Life. Berkeley: University of California Press. 
Bracken, Patrick und Celia Petty (Hg.) 1998. Rethinking the Trauma of War. London, New York: Free Association Press.

Brassett, James, Stuart Croft und Nick Vaughan-Williams 2013. Introduction. An Agenda for Resilience Research in Politics and International Relations. Politics (33.4): 221-228.

Bonß, Wolfgang 2015. Karriere und sozialwissenschaftliche Potenziale des Resilienzbegriffs. In: Martin Endreß und Andrea Maurer (Hg.). Resilienz im Sozialen. Wiesbaden: Springer Fachmedien: 15-31.

Collier, Stephen J. und Andrew Lakoff 2015. Vital Systems Security. Reflexive Biopolitics and the Government of Emergency. Theory, Culture E Society (32.2): 19-51.

Davis, Tracy C. 2007. Stages of Emergency. Cold War Nuclear Civil Defense. Durham: Duke University Press.

Dörre, Klaus, Stephan Lessenich und Hartmut Rosa 2009. Soziologie - Kapitalismus - Kritik. Frankfurt am Main: Suhrkamp.

Dynes, Russel R. und Enrico L. Quarantelli 1975. The Delivery of Mental Health Services in the Xenia Tornado. Columbus, OH (Miscellaneous Report 14).

Dynes, Russell R. und Enrico L. Quarantelli 1968. What Looting in Civil Disturbances really means. Society (5.6): 9-14.

Eckhart, Wolfgang U. 2005. Kriegsgewalt und Psychotrauma im Ersten Weltkrieg. In: Günter H. Seidler und Wolfgang U. Eckart (Hg.). Verletzte Seele. Möglichkeiten und Perspektiven einer historischen Traumaforschung. Gießen: Psychosozial Verlag: 85-107.

Evans, Brad und Julian Reid 2014. Resilient Life. The Art of Living Dangerously. Cambridge UK, Malden Mass: Polity Press.

Fischer-Homberger, Esther 1972. Die Büchse der Pandora. Der mythische Hintergrund der Eisenbahnkrankheiten des 19. Jahrhunderts. Sudhoffs Archiv (56): 297-317.

Foucault, Michel 1994. Das Subjekt und die Macht. In: Hubert L. Dreyfus und Paul Rabinow (Hg.) Michel Foucault. Jenseits von Strukturalismus und Hermeneutik. 2. Aufl. Weinheim: Beltz Athenäum: 243-261.

Foucault, Michel 2004a. Geschichte der Gouvernementalität I. Sicherheit, Territorium, Bevölkerung. Vorlesung am Collège de France 1977-1978. Frankfurt am Main: Suhrkamp.

Foucault, Michel 2004b. Geschichte der Gouvernementalität II. Die Geburt der Biopolitik. Vorlesung am Collège de France 1978-1979. Frankfurt am Main: Suhrkamp.

Fritz, Charles E. und Eli S. Marks 1954. The NORC-Studies of Human Behavior in Disaster. Journal of Social Issues (3): 26-41.

Fritz, Charles E. und Harry B. Williams 1957. The Human Being in Disasters. A Research Perspective. The Annals of the American Academy of Political and Social Science (309.1): 4251.

Fritz, Charles E. 1961. Disaster. In: Robert Merton und Robert A. Nisbet (Hg.). Contemporary Social Problems. An Introduction to the Sociology of Deviant Behavior and Social Disorganization. New York: Harcourt, Brace and World Inc.: 651-694.

Goltermann, Svenja 2009. Die Gesellschaft der Überlebenden. Deutsche Kriegsheimkehrer und ihre Gewalterfahrungen im Zweiten Weltkrieg. München: Deutsche Verlagsanstalt.

Harrington, Ralph 2003. On the tracks of trauma. Railway spine reconsidered. Social History of Medicine (16.2): 209-223.

Hofer, Hans-Georg 2012. Gewalterfahrung, „Trauma“ und psychiatrisches Wissen im Umfeld des Ersten Weltkriegs. In: Helmut Konrad et. al. (Hg). Terror und Geschichte. Köln, Wien: Böhlau: 205-221.

Kaufmann, Doris 1999. „Widerstandsfähige Gehirne“ und „kampfunlustige Seelen“. Zur Mentalitäts- und Wissenschaftsgeschichte des Ersten Weltkriegs. In: Michael Hagner (Hg.). Ecce Cortex. Beiträge zur Geschichte des modernen Gehirns. Göttingen: Wallstein: 205-223.

Fassin, Didier und Richard Rechtman 2009. The Empire of Trauma. An Inquiry into the Condition of Victimhood. Princeton: Princeton University Press.

Höhler, Sabine 2014. Resilienz. Mensch - Umwelt - System. Eine Geschichte der Stressbewältigung zwischen Erholung und Selbstoptimierung. Zeithistorische Forschungen/Studies in Contemporary History (11.3): 425-443.

Howell, Alison 2012. The Demise of PTSD. From Governing through Trauma to Governing Resilience. Alternatives: Global, Local, Political (37.3): 214-226.

Kwon, Heonik 2012. Rethinking Traumas of War. South East Asia Research (20.2): 227-237. 
Moore, Harry Estill und H. J. Friedsam 1959. Reported Emotional Stress Following a Disaster. Social Forces (38. 2): 135-139.

Neocleous, Mark 2012. „Don’t Be Scared, Be Prepared’. Trauma-Anxiety-Resilience. Alternatives: Global, Local, Political (37.3): 188-198.

Neria, Yuval, Sandro Galea und Fran H. Norris (Hg.) 2009. Mental Health and Disasters. Cambridge: Cambridge University Press.

Koch, Ulrich 2014. Schockeffekte. Eine historische Epistemologie des Traumas. Zürich: Diaphanes.

Lasch, Christopher 1979. The Culture of Narcissism. American Life in an Age of Diminishing Expectations. New York, London: Norton.

Lifton, Robert Jay 1973. Home from the War. Vietnam Veterans-Neither Victims nor Executioners. New York: Simon \& Schuster.

Lifton, Robert Jay und Eric Olson 1976. The Human Meaning of Total Disaster. The Buffalo Creek Experience. Psychiatry (39): 1-18.

Luckhurst, Roger 2013. The Trauma Question. New York, London: Routledge.

Lerner, Paul 1996. „Ein Sieg deutschen Willens“. Wille und Gemeinschaft in der deutschen Kriegspsychiatrie. In: Wolfgang U. Eckart und Christoph Gradmann (Hg.). Die Medizin und der Erste Weltkrieg. Pfaffenweiler: Centaurus: 85-109.

Lerner, Paul 2003. Hysterical Men. War, Psychiatry, and the Politics of Trauma in Germany, 18801930. Ithaca: Cornell University Press.

Leys, Ruth 2000. Trauma. A Genealogy. Chicago: University of Chicago Press.

Maxwell, Krista 2014. Historicizing Historical Trauma Theory. Troubling the Trans-Generational Transmission Paradigm. Transcultural Psychiatry (51.3): 407-435.

Micale, Mark und Paul Lerner (Hg.) 2001. Traumatic Past. History, Psychiatry, and Trauma in the Modern Age, 1870-1930. Cambridge: Cambridge University Press.

Morris, Andrew J. F. 2011. Psychic Aftershocks. Crisis Counseling and Disaster Relief Policy. History of Psychology (14.3): 264-286.

Pomberger, Markus et al. 2015. Chancen des Resilienzbegriffs für eine soziologische Armutsforschung. In: Martin Endreß und Andrea Maurer (Hg.). Resilienz im Sozialen. Wiesbaden: Springer Fachmedien: 265-294.

Quarantelli, Enrico L. 1979. The Consequences of Disaster for Mental Health. Conflicting Views. Columbus, OH (Preliminary Paper 62).

Quarantelli, Enrico L. 1984a. An Overview of Research on PTSD in Survivors of Disasters. Columbus, OH (DRC Working Paper 72).

Quarantelli, Enrico L. 1984b. An Assessment of Conflicting Views on the Consequences of Community Disasters for Mental Health. Columbus, OH (DRC Preliminary Paper 89).

Rieff, Philipp 1966. The Triumph of the Therapeutic. Uses of Faith after Freud. New York: Harper \& Row Publishers.

Pupavac, Vanessa 2001. Therapeutic Governance. Psycho-social Intervention and Trauma Risk Management. Disasters (25.4): 358-372.

Schmitz, Helmut und Annette Seidel Arpaci (Hg.) 2011. Narratives of Trauma. Discourses of German Wartime Suffering in National and International Perspective. Amsterdam, New York: Rodopi.

Shephard, Ben 2000. A War of Nerves. Soldiers and Psychiatrists 1914-1994. London: J. Cape.

Stehrenberger, Cécile Stephanie 2014: Systeme und Organisationen unter Stress. Zur Geschichte der sozialwissenschaftlichen Katastrophenforschung (1949-1971). Zeithistorische Forschungen/Studies in Contemporary History (11): 406-424.

Stehrenberger, Cécile Stephanie 2016 (forthcoming): Katastrophen-Fall-Wissen. Zur Geschichte der Sozialwissenschaftlichen Katastrophenforschung. Itinera: Beiheft zur Schweizerischen Zeitschrift für Geschichte.

Summerfield, Derek 2001. The Invention of Post-Traumatic Stress Disorder and the Social Usefulness of a Psychiatric Category. British Medical Journal (322): 95-98.

Summerfield, Derek 2002. Effects of War. Moral Knowledge, Revenge, Reconciliation, and Medicalised Concepts of „Recovery“. Britisch Medical Journal (325): 1105-1107.

Thomas, Gregory M. 2009. Treating the Trauma of the Great War. Soldiers, Civilians and Psychiatry in France, 1914-1940. Baton Rouge: Louisiana State University Press.

Taylor, Verta A. 1976. Delivery of Mental Health Services in Disasters. The Xenia Tornado and some Implications. Columbus, OH: Ohio State University. 
Tierney, Kathleen J. und Barbara Baisden 1977. Crisis Intervention Programs for Disaster Victims. A Source Book and Manual for Smaller Communities. Columbus, OH (Miscellaneous Report 22).

Winter, Jay 2000. Shellshock and the Cultural History of the Great War. Journal of Contemporary History (35.1): 7-11.

Withuis, Jolande und Annet Mooij (Hg.) 2010. The Politics of War Trauma. The Aftermath of World War II in Eleven European Countries. Amsterdam: Aksant.

Wolfenstein, Martha 1957. Disaster. A Psychological Essay. London: Routledge \& K. Paul.

Young, Allan 1997. The Harmony of Illusions. Inventing Post-Traumatic Stress Disorder. Princeton NJ: Princeton University Press.

Cécile Stephanie Stehrenberger

Max-Weber-Kolleg für sozial- und

kulturwissenschaftliche Studien

Universität Erfurt

Postfach 900221

99105 Erfurt

Germany

E-Mail: cecile.stehrenberger@uni-erfurt.de 